Linguistique, littérature, didactique

191-192 | 2021

Théâtre contemporain : Écritures et Représentations

\title{
La dramaturgie sans action : Maeterlinck, Beckett, Lagarce
}

Jean-Paul Dufiet

\section{OpenEdition}

Journals

Édition électronique

URL : https://journals.openedition.org/pratiques/11110

ISSN : 2425-2042

Éditeur

Centre de recherche sur les médiations (CREM)

Référence électronique

Jean-Paul Dufiet, «La dramaturgie sans action : Maeterlinck, Beckett, Lagarce », Pratiques [En ligne], 191-192 | 2021, mis en ligne le 15 décembre 2021, consulté le 16 décembre 2021. URL : http:// journals.openedition.org/pratiques/11110

Ce document a été généré automatiquement le 16 décembre 2021.

(c) Tous droits réservés 


\title{
La dramaturgie sans action : Maeterlinck, Beckett, Lagarce
}

\author{
Jean-Paul Dufiet
}

\section{Introduction}

1 D'après La Poétique d'Aristote (1980, p. 53-57), le théâtre, en particulier le drame à la suite de la tragédie, représente une action dialoguée au présent qui exprime un conflit (Pavis, 1980, p. 86-89) entre des individus. La résolution du conflit à travers une «fable » transforme les relations entre ces individus et la situation initiale du monde représenté. Dans son langage, P. Szondi parle d'un événement interhumain, d'une « intersubjectivité » qui entraîne des « décisions ${ }^{1}$ » de la part des personnages (Szondi, 1983 [1956], p. 13). À la différence du théâtre historique ou épique, le drame possède un caractère " primaire » (ibid., p. 15), au sens où il ne trouve son origine qu'en lui-même. Cette conception, qui a été l'objet de si nombreux commentaires et qui demeure toujours une référence incontournable pour saisir l'écriture dramatique passée et contemporaine, est entrée en crise à la fin du XIX siècle (ibid.; Sarrazac, 2012). Dans toute l'Europe, l'action et le conflit, qui forment un couple solidaire, ne sont plus considérés, par de nombreux auteurs, comme le moteur structurant du théâtre (Sarrazac, 2015, p. 155-157). Dans le contexte francophone et à la suite de M. Maeterlinck (1862-1949) qui a développé la théorie d'un théâtre statique, cette idée s'est répandue et s'est toujours plus renforcée tout au long des $\mathrm{Xx}^{\mathrm{e}}$ et $\mathrm{XxI}$ siècles. La pièce sans action, ou la pièce dont l'action est squelettique, est devenue un lieu commun de l'écriture dramatique contemporaine.

2 En langue française, S. Beckett ${ }^{2}$ (1906-1989) et J.-L. Lagarce (1957-1995), en particulier, prolongent l'orientation de $\mathrm{M}$. Maeterlinck et scandent, de manière large, ce siècle et demi d'écriture dramatique. La mise en cause de l'action s'inscrit maintenant dans une durée longue et ces trois dramaturges appartiennent, avec des différences, à la même lignée. Nous voudrions donc examiner J'étais dans ma maison et j'attendais que la pluie vienne $^{3}$ de J.-L. Lagarce(1994) à la lumière de Pelléas et Mélisande ${ }^{4}$ de M. Maeterlinck 
(1892) et de En attendant Godot ${ }^{5}$ de S. Beckett (1952). De quelle manière l'action est-elle réduite ou évacuée dans ces trois textes? Quelles sont les conséquences sur le dialogue et la parole qui sont le vecteur de l'action et qui pourtant persistent en l'absence cette dernière ? Enfin, qu'en est-il de la théatralité de ces pièces ? En particulier de celle de J.-L. Lagarce, dès lors qu'elles n'ont plus comme finalité de représenter et de porter à terme, selon les principes de la mimésis, un conflit qui définit une action. Comment les textes se désignent-il eux-mêmes comme théâtre? En dehors de certaines caractéristiques typographiques et du désir des auteurs d'être représentés sur scène, qu'est-ce qui se substitue à l'action dans la totalité du texte et qui justifie que ces textes appartiennent au genre dramatique?

\section{Des pièces sans action}

\section{Le personnage aboulique}

3 La forme du théâtre de M. Maeterlinck dépend très directement de son ontologie générale. L'agent humain de M. Maeterlinck est prisonnier de son «impuissance existentielle » (Szondi, 1983 [1956], p. 49). M. Maeterlinck nie d'abord le sujet agissant, de sorte que l'action dramatique disparaît comme une conséquence logique de l'absence de protagoniste actif. L'action s'efface en raison de l'apparition d'actants humains abouliques. Dans $P$. et $M$., il reste un schéma très stéréotypé d'une rivalité amoureuse et d'un amour impossible; la particularité de ce schéma est qu'il s'accomplit, apparemment, de lui-même, sans que la volonté des actants (Pelléas et Mélisande) ne semble y prendre part véritablement. P. et M. crée un espace imaginaire et poétique qui est inaccessible au sens commun, qui laisse deviner un profond et important territoire de l'existence humaine qui échappe tout à la fois à la raison et au visible, et qui se soustrait, du point de vue dramaturgique, à la mimésis aristotélicienne. Les personnages ne sont pas les sujets d'une action, mais ils sont les objets de forces mystérieuses et inconnues qui les dominent et les manipulent malgré eux. Ce choix est conditionné par la philosophie et l'esthétique symbolistes. Sans reposer sur les mêmes présupposés philosophiques, plusieurs pièces de J.-L. Lagarce présentent des personnages qui sont impuissants et sans volonté, comme les cinq femmes de JMM qui n'ont fait qu'attendre, ou bien qui sont hors d'état d'exprimer une quelconque volonté, comme le jeune Frère (ou Fils) ${ }^{6}$ toujours dans JMM, dont le spectateur n'entend parler qu'au moment de l'épuisement final de sa volonté, puisqu'il revient pour mourir. Entre les personnages de M. Maeterlinck et ceux de J.-L. Lagarce, on trouve Vladimir et Estragon qui tout comme les cinq femmes de JMM ne font qu'attendre un certain Godot, et qui renoncent de manière systématique à leurs plus légères velléités d'agir. L'absence d'action est comme thématisée par le refus de décider quoi que ce soit et par l'impossibilité de mobiliser une volonté libre. J.-P. Sarrazac (2012, p. 183-241) y voit une catégorie poétique qu'il nomme "l'impersonnage ». Le profil de ce type de personnage rend toute action impossible. Toutefois, l'orientation de certaines autres catégories de la poétique théâtrale contribue également à composer des pièces sans action. La nature de la situation dans laquelle sont plongés les personnages en fait partie. 


\section{La situation d'attente}

4 L'absence de volonté s'accorde fort bien avec une situation dans laquelle un personnage est absent et est attendu par d'autres. Attendre quelqu'un justifie la passivité de ceux qui attendent et qui ne font rien d'autre qu'attendre. Ces figures vivent pour le seul moment où l'absent devrait (ré)apparaître. Il y a une solidarité sémantique et structurelle entre l'aboulie et l'attente, qui est par excellence une situation de nonaction. Avec un schéma d'attente, le texte donne fréquemment force aux événements qui se déroulent en dehors de la scène sans que les personnages qui sont en scène ne veuillent agir sur ces événements, qui de toute manière restent hors de leur portée. Les personnages sans volonté sont plongés dans une situation qui les met dans l'incapacité de prendre la "décision » qui constituerait le déclencheur d'une action. C'est ce qui caractérise $E A G$, dans laquelle l'inaction est enfantée par une situation d'attente. Dans $J M M$, l'inaction est plus marquée encore que dans EAG, puisqu'entre le début et la fin de $J M M$, à la lettre, rien n'advient, alors que dans EAG, les apparitions de Lucky et Pozzo, ainsi que celle du Garçon, perturbent un tant soit peu l'attente de Vladimir et Estragon, même si elles ne la transforment pas véritablement. En revanche, dans JMM, J.L. Lagarce exploite de manière très différente la situation d'attente, en la tordant, puisque celui qui était attendu vient à peine de revenir lorsque la pièce commence.

5 Le premier monologue de JMM nous fait comprendre qu'un homme jeune sans prénom, Frère-Fils de cinq femmes, est réapparu pour agoniser dans la maison familiale où il avait vécu jusqu'à la fin de son adolescence. Il en était parti à la suite d'une de ses nombreuses et violentes disputes avec son père, mort désormais. Les cinq femmes l'ont attendu, pendant toutes les années de son absence, sans rien faire d'autre que l'attendre.

6 Le titre, J'étais dans ma maison et j'attendais que la pluie vienne, telle une phrase incomplète, laisse espérer qu'une transformation est possible, puisque l'imparfait semble lui aussi attendre un passé simple destiné à interrompre la continuité (Kuntz, 2008, p. 11-28). Ce titre prépare l'arrivée d'une subordonnée temporelle qui d'ailleurs est effectivement prononcée dès les premières phrases du monologue : «et c'est à ce moment exact qu'il apparut, et que je le vis » (Lagarce, 1997, p. 9). La pièce, comme dans toute la dramaturgie traditionnelle, annonce un fait perturbateur qui, dès le début de la représentation, devrait modifier l'état présent du monde dans lequel le spectateur entre :

J'attendais la pluie, j'espérais qu'elle tombe,

j'attendais, comme, d'une certaine manière, j'ai toujours attendu,

j'attendais et je le vis,

j'attendais et c'est alors que je le vis, celui-là, le jeune

frère, prenant la courbe du chemin et montant vers la

maison, j'attendais sans rien espérer de précis et je le

vis revenir, j'attendais comme j'attends toujours,

depuis tant d'années, sans espoir de rien. (ibid.)

7 Cette attente est enfin comblée, dès le début, à l'exact opposé de la construction de $E A G$; et ce retour devrait transformer radicalement la situation des cinq femmes et les faire sortir, enfin, de leur immobilisme de pierre. En fait, c'est très exactement tout ce qui ne se produira pas. J.-L. Lagarce liquide ce programme dramatique potentiel qu'il suggère dans le titre. En quelque sorte, le retour du Frère-Fils, ne fait que souligner l'immobilisme radical des cinq personnages féminins, et mettre au premier plan ce que 
J.-L. Lagarce lui-même appelle «le statisme des vies » (Lagarce, 2011, p. 132). L'Aînée des cinq femmes poursuit ainsi son monologue :

Celui-là, le jeune frère, revenu de ses guerres, je le vis

enfin et rien ne changea en moi,

j'étais étonnée de mon propre calme, aucun cri comme

j'avais imaginé encore et comme vous imaginiez

toutes, toujours, que j'en pousserais, que vous en

pousseriez, notre version des choses,

aucun hurlement de surprise ou de joie,

rien. (ibid., 1997, p. 10)

Elle expose rétrospectivement qu'elle n'éprouve aucune des réactions de joie et d'enthousiasme qu'elle-même et les autres femmes avaient imaginées ressentir au moment de ce retour. Elle comprend à l'instant du retour combien elle est engluée dans sa propre existence : «Je le voyais marcher vers moi et je songeais qu'il revenait et que rien ne serait différent, que je m'étais trompée » (ibid.). Le retour ne met pas un terme à l'attente; au contraire, il en révèle la radicalité et il la cristallise. L'attente dans JMM révèle un théâtre de la famille qui expose deux voies d'échec. Les femmes qui restent au foyer s'y consument dans le ressassement, mais celui qui le quitte se perd, y revient au seuil de la mort, incapable de rien donner à celles qui sont restées.

Si le départ du Jeune Homme, fut un drame dans la vie des femmes, avec le temps il est devenu le drame de leur vie (Sarrazac, 2012, p. 65-99). On comprend que leur vie à venir sera identique à leur vie passée.

\section{Attendre}

Le personnage du Frère-Fils n'est pas un agent transformateur de la situation et il n'inaugure pas un recommencement de la vie. L'attente ne connaît donc pas d'épilogue et l'enlisement des cinq femmes, dans l'adverbe "toujours» s'aggrave. Au plan dramaturgique de la monstration, le jeune homme n'apparaît jamais en scène et le moment du retour est antérieur au début de la pièce; les spectateurs en sont donc privés. En ce sens, cette image d'Épinal de la réunion de la famille n'a pas plus de réalité mimétique que l'invisibilité de Godot ou que la rencontre entre Pelléas et Mélisande à la scène $5 \mathrm{du} \mathrm{III}^{\mathrm{e}}$ acte. On se rappelle que cette célèbre scène de M. Maeterlinck n'est que racontée par Yniold et qu'elle est inaccessible à Golaud et au spectateur. La dramaturgie de l'inaction organise fréquemment l'invisibilité de certains personnages, même quand ils pourraient être en scène.

11 Le retour du Frère-Fils dans JMM est donc dit au passé simple, y compris pour marquer combien ce moment n'a aucun effet sur le présent. Celui qui était attendu est enfin revenu mais la signification de son retour est peut-être pire que celle des fausses arrivées de Godot. D'ailleurs, rappelons qu'EAG débute par le retour d'Estragon que Vladimir attendait. Estragon, qui croyait être parti pour toujours (Beckett, 1952, p. 9), a dormi, au cours de son bref éloignement, dans un fossé et n'a récolté que des coups (ibid., p. 10). Ce début d'EAG n'offre-t-il pas un micro-schéma que JMM développe et intensifie à l'extrême ? Le Frère-Fils est allé prendre des coups, seul, hors du cocon familial et il y revient auprès de celles qui l'ont attendu, mais dans un très piteux état et pour agoniser.

Grâce au motif de l'attente, J.-L. Lagarce construit une immobilité dramatique dont il écrase les réserves d'imaginaire. Dans JMM, il y a deux drames qui sont sans interaction 
réciproque : celui du retour et de la mort du Frère-Fils, et celui de l'attente passée et future des cinq femmes. Les cinq femmes commentent leur attente passée, auscultent leur réaction présente, et se préparent à une attente sans objet ni terme. Comme le dit J.-P. Sarrazac (2012, p. 77-85), la vie est réduite à l'« infradramatique », qui ne se nourrit ni de héros, ni de conflit. C'est le drame de la non vie, de la vie insuffisante. L'attente est devenue un état de l'être, une longue mélancolie, comme si à force d'attendre leur Frère-Fils "sans espoir de rien » (Lagarce, 1997, p. 9) les femmes avaient transformé leur situation d'attente en état intérieur, leur contingence en essence, sans bien évidemment que cette transformation ne puisse être assimilée à une "décision " qui exprimerait la volonté des personnages et qui accomplirait ainsi leur "réalisation dramatique » (Szondi, 1983 [1956], p. 13).

13 Mais en fait, cette famille avait-elle jamais commencé à vivre authentiquement? L'inaction ne serait-elle pas sa manière, depuis toujours, d'être ensemble et de se protéger des coups du monde, comme le font Estragon et Vladimir. Ne vaut-il pas mieux attendre Godot, ou un familier parti vers l'aventure, que de se risquer à la vie? «Est-ce que je n'ai pas toujours attendu? » dit une des femmes (Lagarce, 1997, p. 13). En somme, le départ du jeune homme pourrait bien révéler qu'au commencement était l'attente. Ces cinq femmes ne sont pas en mesure, comme elles disent, de «bouger d'elles-mêmes» (ibid., p. 8); elles prolongent la vie dans une sorte de démenti à A. Schopenhauer, comme si la vie elle-même n'avait aucun intérêt à vivre. J.-L. Lagarce, sur les traces de M. Maeterlinck et de S. Beckett, transforme une situation contingente en ontologie. Toute la parole des cinq femmes est concentrée en une seule journée pour dire leurs années d'attente dont personne ne sait donner le compte. Les personnages sont entièrement enveloppés dans un temps immobile et répétitif et nul ne sait quelle heure il est dans le temps de cette famille. Le jour du retour est la date dont chaque femme se rappellera comme le jour du retour sans retrouvailles. Alors que dans P. et $M$. et dans EAG une succession de faits scande une durée, dans JMM toutes les temporalités sont enfermées dans le présent de la parole. L'énonciation fictionnelle dit le présent au passé : «Aujourd'hui, en ce jour précis, je pensais à cela, en ce jour précis, je pensais ${ }^{7}$ à cela " (ibid.). Arrive un imparfait là où on attendrait un passé composé ou un présent. Mais le futur aussi est absorbé par l'énonciation fictionnelle immédiate : « Il nous quitte, il nous laisse, c'est nous qui toujours, là, toutes les cinq, c'est nous, toutes, qui l'attendrons, toutes ces années perdues, restées bloquées, épuisées, là » (ibid., p. 38). Le moment du trauma indélébile du départ du Frère-Fils est comme l'origine de toutes les énonciations des personnages. Le présent ne se présente plus comme tel, dans sa nouveauté, il ne se distingue plus des moments précédents parce que rien ne sépare les instants les uns des autres, pas même le retour du Frère-Fils. Les cinq femmes sacralisent le moment du départ que le moment du retour n'effacera pas; il en résulte que toutes les temporalités se confondent et que les discours des personnages sont à «l'écart du temps" (Bident, 2010, p. 60).

14 Cette impossibilité à distinguer les temporalités rapproche la pièce de J.-L. Lagarce d'une scène mentale. Comme on le sait, $\mathrm{M}$. Maeterlinck a souvent composé son théâtre statique comme un rêve, et $P$. et $M$. procède par images oniriques mystérieuses ${ }^{8}$. Dans cette lignée, JMM ressemble au scénario mental d'un jeune homme qui rêve que sa famille pense à lui. La pièce s'apparente à un fantasme adolescent, qui n'est pas sans narcissisme. Celui qui est parti imagine ce que sa famille, limitée aux femmes, pense de lui pendant son absence, et ce qu'elle dirait de lui à son retour, comme s'il craignait d'avoir perdu leur amour et d'être oublié (Mirjol, 2010, p. 71), lui qui a si bien oublié les 
autres. Ce jeune homme rêve d'être l'objet d'intérêt non seulement principal, mais unique, de celles qu'il a laissées. Dans ce « theatrum mentis » (Sarrazac, 2008, p. 277), il donne voix à son désir d'être attendu et il réduit toute la vie des cinq femmes au seul moment très étiré d'une attente illimitée. Car si dans la fiction, le Frère-Fils obsède les cinq femmes, en revanche dans la perspective autobiographique (Valéro, 2008, p. 237-252) où s'inscrit l'essentiel de la production dramatique de J.-L. Lagarce, n'est-ce pas en fait le groupe des cinq femmes qui occupe l'esprit du Frère-Fils et parle en lui ?

\section{La parole} direct entre l'action et la parole. La parole existe au théâtre en tant que force performative. C'est l'intersubjectivité qui introduit l'action et le conflit grâce au dialogue comme forme de l'échange interhumain (Szondi, 1983 [1956], p.14). Par conséquent, construire un théâtre sans action, ce n'est pas seulement évider la fable de tout conflit structurant, c'est aussi dévitaliser la parole agissante qui règne dans la poétique classique.

Même si M. Maeterlinck, S. Beckett - moins que les autres auteurs - et J.-L. Lagarce conservent formellement un dialogue organisé par une distribution de tours de parole, ils n'en construisent pas pour autant une authentique relation interhumaine (ibid., p.63) qui «porte le drame». La disparition de l'action s'accompagne d'une transformation du dialogue classique, au point que dans P. et M., EAG et JMM, les échanges verbaux ne sont plus guidés par des structures verbales d'antagonisme entre les personnages.

17 En réalité, nos trois pièces sans action offrent des formes de dialogue communes et différentes. D'un côté, la parole est contaminée par le silence et le non-dit (P. et M., EAG, $J M M$ ) et d'un autre côté, les personnages de JMM, et eux seulement, sont dévorés par une forme de logorrhée dont J.-L. Lagarce s'est fait un véritable stylème d'écriture.

\section{Le silence et le non-dit}

À rebours de l'action dramatique et du dialogue performatif, M. Maeterlinck délimite dans la parole un territoire de "l'inexprimable » qui est étranger à la dramaturgie classique (ibid., p.13). Même s'il continue de faire parler ses personnages, le dramaturge belge récuse le dialogue dans son essence même. D'ailleurs, comme beaucoup de symbolistes, il ne fait pas confiance à la langue, et il ne l'utilise que par défaut, faute de disposer d'une autre forme de langage. Cette conception explique le choix du silence comme véritable expression des personnages. On en rencontre un exemple important dans le moment d'échange le plus intense entre Pelléas et Mélisande, à la scène 5 de l'acte III, par l'intermédiaire d'Yniold: "Ils [Pelléas et Mélisande ${ }^{9}$ ne se parlents pas ", "Ils ne disent rien" alors qu'ils sont dans une chambre, seuls ; "Ils regardent la lumière ", "Ils ne ferment pas les yeux ", "Ils ne bougent pas» (Maeterlinck, 2012, p. 59). Ce n'est pas par les mots que Pelléas et Mélisande communiquent ${ }^{10}$. En un sens, le silence devrait exprimer tout ce que les langues naturelles ne pourront jamais formuler, et le cœur du tragique, tel que M. Maeterlinck (1917, p. 9-25) le conçoit et l'expose dans Le Trésor des humbles se dessine autour des impuissances de l'expression verbale. Les personnages de Pelléas et de 
Mélisande se réfugient dans un monde où l'absence de mots tend à être la forme d'expression parfaite de ce que M. Maeterlinck (ibid., p.11) appelle le «dialogue des âmes ». Il condense cette conviction en une formule tout à la fois paradoxale et radicale : «Dès que nous avons quelque chose à dire, nous devons nous taire » (ibid.).

On pourrait dire que $\mathrm{S}$. Beckett, avec des présupposés philosophiques et des finalités esthétiques différents, a réalisé le projet de $\mathrm{M}$. Maeterlinck en créant si souvent des personnages qui dérivent du laconisme au mutisme. Puisque pour S. Beckett être au monde est un pur désastre, tout se concentre dans l'union de l'origine et de la disparition humaines. Pour l'auteur d'Acte sans paroles (Beckett, 1956), il n'y a guère à dire ; selon une formule bien connue il n'y a qu'à « dire l'échec à dire ».

Dans JMM, seul le personnage du Frère-Fils est privé de la parole par le dispositif dramatique. Le spectateur n'assiste à aucun de ses échanges, s'il y en a eu, avec les cinq femmes. On peut imaginer que ces dernières auraient aimé entendre un récit de voyage et d'expérience, des confidences aussi. Il y a comme une odyssée à faire entendre. Pas même une bribe de discours rapporté qui aurait été prononcé par le Frère-Fils ne parvient au spectateur. La parole des femmes se déploie autour de celui qui se tient hors du verbe.

21 Toutefois, dans les pièces sans action, la transformation du dialogue en échange non performatif, ne s'effectue pas seulement grâce à une valorisation du silence complet ; elle se réalise également par une incomplétude du dit. Les dialogues sont défaillants parce que sciemment composés avec des manques importants. Les répliques se contentent d'évoquer les événements, de les dire imparfaitement. Qu'on se rappelle, par exemple, que Mélisande ne raconte son histoire que de manière très embryonnaire, lorsqu'elle rencontre Golaud. Golaud lui-même, après la mort de Pelléas et pendant l'agonie de Mélisande, déclare, à l'instar du moment de l'anagnorisis aristotélicienne, qu'il a compris son propre destin; jamais pourtant, à l'inverse des personnages classiques, il ne révèle la teneur de ce qu'il a saisi. On peut ajouter d'autres exemples. En effet, Vladimir et Estragon ne rapportent, avec une extrême confusion, que quelques paroles incertaines de Godot. Dans JMM, les cinq femmes répètent qu'elles n'ont pas d'histoire, et aucune d'elles ne révèle le motif de cette dispute, entre le père et le FrèreFils, qui provoqua la cassure de l'unité familiale : « Lorsque le père le chassa, le mit à la porte, vous ne dites jamais cela, vous gardez votre secret » (Lagarce, 1997, p. 29). Seule La Plus Jeune évoque " [de] la haine et la lueur du crime » (ibid., p. 30). Mais quoi qu'il en soit, aucune raison claire ne transpire. Le motif est tu, et ce silence appartient aux importants non-dits de la pièce, à la violence familiale qui reste muette et ne sort pas des murs de la maison. Paradoxe d'une dramaturgie qui s'enroule[..] autour d'un moment unique mémoriel » (Sarrazac, 2012, p. 171), fait de mots, et que le spectateur connaît seulement de manière partielle. Ces mots prononcés par le père dans le passé ont chassé le Frère-Fils et ont exprimé une force performative qui n'a plus cours. Ils avaient provoqué une « décision » (Szondi, 1983 [1956], p. 14). C'est comme si J.-L. Lagarce avait fictionnalisé la fin de la force pragmatique des paroles. Ces mots tus, qui ont orienté effectivement et fortement le destin de la famille, sont refoulés.

En raison de son rôle dans la dramaturgie aristotélicienne, le dialogue ne peut donc pas ne pas être affecté par la disparition de l'action. La parole performative, dont il est porteur, est remplacée dans $P$ et $M, E A G$, et JMM par le silence, par l'incomplétude verbale et par le non-dit. Cette dramaturgie elliptique exige que le spectateur accepte que des questions, que le texte lui-même suscite, restent sans réponse. Le dialogue n'a 
pas alors seulement la fonction d'exposer le sens, il a également celle de mettre en évidence la privation de sens.

\section{La parole solitaire et surabondante}

Toutefois la parole chez J.-L.Lagarce se distingue radicalement de celle de M. Maeterlinck et de S. Beckett. On sait combien le théâtre de S. Beckett va jusqu'à une réduction maximum du texte parlé, réduit à des didascalies muettes, comme si l'écriture elle-même était engloutie par le silence.

Dans JMM, le silence et le non-dit sont entourés d'une dépense verbale à la fois solitaire et très copieuse. Au fond, chez J.-L. Lagarce, l'épuisement de l'action dramatique ouvre la parole. Dans JMM, chaque femme locutrice dit les vicissitudes de son existence, en se parlant autant à soi-même ou au spectateur qu'aux autres personnages. Le sujet fictionnel, parce qu'il est libéré de toute action, bénéficie d'une capacité d'examen de soi grâce à laquelle il met à jour, plus ou moins directement et précisément, son identité. Le théâtre contemporain, et en particulier celui de J.-L. Lagarce, privilégie souvent une parole solitaire, que ce soit monologue ou soliloque, plutôt qu'un enchaînement de répliques qui se répondent. Le dialogue d'action est remplacé par des formes repliées de la parole, qui sont propices au développement de l'intériorité. En conséquence de quoi, la collaboration des énonciations fictionnelles constitue plus un chœur que la mimésis d'une conversation. Dans JMM, les cinq femmes composent un ensemble polyphonique (Jolly, 2008, p. 222) dont les membres sont unis par une ou deux thématiques; au plan discursif, la convergence énonciative enveloppe la discordance des opinions. Les voix ne s'opposent plus directement comme dans le dialogue d'action, mais elles se conjuguent, plus ou moins intensément, dans la différence.

Dans de nombreuses pièces de J.-L. Lagarce, les personnages locuteurs ne sont pas seulement des adeptes du soliloque, ils sont également très prolixes, au point de submerger le spectateur par l'abondance de leur parole. Les cinq femmes de JMM partagent cette caractéristique. Les personnages sont tous étonnamment diserts. Mais cette prolixité n'est pas l'effet d'une faconde de caractère, partagée par les cinq femmes. En réalité, l'abondance verbale hors norme de chaque personnage est due aux modalités complexes, et communes, de leur acte de parler.

Leur parole est faite de multiples figures de rhétorique, très proches les unes des autres à l'intérieur de la phrase, et très voisines par leur fonction et leur signification. Se succèdent donc dans le texte : des répétitions, des reformulations, des ajustements, des reprises, des épanorthoses, des nuances, et dans d'autres cas des réticences, des rectifications, des paremboles (Sarrazac, 2008, p. 284-285), des incises aussi, et parfois des digressions assez longues. C'est une parole qui ne sait pas aller droit au but, qui cherche son chemin, et qui semble être en échec constant (ibid., 2015, p. 184). Les femmes ne cherchent pas seulement le mot juste; elles semblent vouloir énoncer tous les mots possibles qui envelopperaient sans reste les diverses facettes de la situation, de l'événement, de la pensée, des sentiments, et même de l'acte de dire. Le sens tend alors à se gélifier sous la pression des trop nombreuses possibilités lexicales, plus ou moins synonymes. La parole s'ajuste moins qu'elle ne bégaie et piétine. Le discours progresse très lentement, en revenant sur son lexique comme les anneaux d'une spirale. Les prédicats se bousculent, le focus arrive en retard, incertain de lui-même. L'axe 
paradigmatique se répand sur l'axe syntagmatique. Des énoncés simples, indépendants et brefs se juxtaposent avec des liens sémantiques, mais sans liens syntaxiques forts comme s'ils s'imposaient au locuteur qui les formule. C'est une sorte d'ébullition verbale; au lieu d'avancer, la parole bouillonne.

Et cette parole, même quand elle est adressée, n'aspire pas au dialogue (Talbot, 2008, p. 258). Elle anticipe souvent des objections par un contre-discours. De la sorte, elle fait droit au point de vue d'autrui tout en le vanifiant, puisqu'il a été intégré et désamorcé ; il devient donc inutile ou superfétatoire. Il en résulte d'ailleurs que les personnages de J.-L. Lagarce parlent souvent à la place des autres. « La prolifération de la parole épuise le champ des réponses possibles de sorte que le destinataire [...] n'a plus qu'à jouer solipsisme contre solipsisme » (ibid., p. 265). Cette forme de parole noie l'espace de l'échange et élimine la possibilité même d'une interaction dramatique. Paradoxalement, J.-L. Lagarce a une conception très aristotélicienne du théâtre, qu'il réalise à rebours des intentions du théoricien grec: ce fleuve de mots auquel il est quasiment impossible de répondre élimine tout dialogue authentique, vecteur d'une action dramatique. JMM repose donc sur une solidarité essentielle entre la forme de la parole et l'absence d'action. La parole est surabondante et dévitalisée, privée de sa force performative qui est sa propriété première au théâtre. Dans le dialogue classique, la performativité naît d'une expression verbale orientée par une finalité. Elle laisse également toute sa place à l'interlocuteur parce qu'elle a besoin de lui pour se réaliser. À l'inverse, la parole de JMM qui revient sur elle-même et se déséquilibre constamment est profondément anti-performative. La reformulation, la variation et la synonymie épuisent la force du dire.

C'est donc une autre fonction de la parole qui se réalise dans JMM. Les cinq femmes, parce qu'elles examinent continuellement les mots qu'elles emploient, ont une conscience métalinguistique aiguë qui se substitue à la performativité de leur dire. Le personnage semble entièrement occupé à évaluer la justesse de ses énoncés. En s'exposant exclusivement comme un parleur et en intervenant constamment sur son propre discours, chaque personnage de JMM affirme ainsi sa présence énonciative contre son impuissance performative et conquiert l'existence dont le prive son inaction dans le réel de la fiction. Dans JMM, le démembrement de l'action fait que la pièce est moins organique, moins soumise à une construction; par conséquent, la parole des personnages est libérée des règles de la composition dramatique. En même temps, la disparition de l'action produit une réduction de la mimésis au profit de la diégesis, au point que le texte devient un objet verbal clos sur lui-même. Alors que chez M. Maeterlinck et S. Beckett la disparition de l'action entraîne une mise en cause de la parole, chez J.-L. Lagarce la présence de la parole en ressort renforcée au détriment de sa performativité.

\section{La théâtralité}

\section{Le métadrame}

Dès lors que l'action est réduite à la portion congrue, ou même qu'elle disparaît, comment les pièces, qui dans la tradition aristotélicienne représentent l'imitation des actions humaines, font-elles désormais théâtre, comment appartiennent-elles au genre dramatique ? En d'autres termes, quelle est leur théâtralité ? 
30 En fixant à son théâtre l'horizon du mutisme et de l'invisible, M. Maeterlinck vise la catégorie de l'irreprésentable, et semble annuler la théâtralité de son théâtre. L'objet même de la scène, c'est ce qui ne peut être ni vraiment dit ni exactement montré. Le dramaturge organise la disparition de son langage dramatique. Il cherche, ou rêve, un impossible langage au bord du sensible, détaché de tout ordre de communication intelligible, et qui soit en mesure de laisser entrevoir l'inexprimable (Dessons, 2016, p. 13). On comprend qu'aucune forme de langage verbal puisse véritablement relever un tel défi. M. Maeterlinck établit son théâtre à la limite de la non représentation, comme s'il le laissait, tel S. Mallarmé, entre les pages du livre.

31 En fait, avec la disparition de l'action et la transformation du dialogue, le théâtre en vient à s'interroger sur lui-même tout en se faisant. Il se tourne alors vers la métathéâtralité, non pas pour jouer sur les effets de l'illusion scénique comme le faisait le théâtre baroque, mais pour questionner les formes et la finalité du langage dramatique.

Contrairement aux dramaturges dont l'écriture exhibe la métathéâtralité de leur texte par un jeu entre différents niveaux de représentation ${ }^{11}$ (Sarrazac, 2012, p. 27), J. L. Lagarce inscrit le métadrame dans la substance de la fiction, dans la situation ellemême. Le Frère-Fils revient dans la maison familiale pour compléter et conclure sa propre histoire, puisqu'elle arrive à son terme. C'est le regard et la parole de celles qui sont restées qui manquent à sa vie qu'il est le seul à connaître. N'est-ce pas pour qu'elles soient obligées de parler de lui qu'il leur apporte son corps agonisant devant la porte de la maison? Ce Frère-Fils, membre de la famille, au bord de la mort dans son lit, ne demande-t-il pas aux cinq femmes d'écrire dans le tressage de leur parole un texte qui appartient aussi aux genres littéraires de l'éloge funèbre et du tombeau ? Il peut, en tant que personnage, accomplir la magie théâtrale d'être ici au présent sans y être, corps caché entièrement voué à ce que l'on dit de lui, dans une position semblable à celle de l'outre-tombe, d'où il provoque la parole, assistant dans sa présence absence à cette cérémonie des adieux. Au plan de l'écriture, en ce sens, la fonction du Frère-Fils de JMM n'a-t-elle pas de fortes similitudes avec celle de Godot? Ces deux «impersonnages» sont absents de la scène, invisibles, et font parler les autres personnages. N'est-ce pas, en vérité, exactement la fonction du dramaturge ? Être toujours présent derrière la parole des locuteurs fictionnels, et n'être visible comme tel à aucun moment. Le Frère-Fils, plus encore que Godot, a tout du dramaturge. Le moment le plus intense de la vie n'est pas celui où on l'a vécu mais celui où ce vivre est raconté.

33 La métathéâtralité se trouve également dans le regard et la parole des personnages féminins qui examinent leurs propres rôles dans la vie. En l'absence d'action, ces personnages s'auscultent, dans la mesure même où ils sont dépourvus de volonté et de projet. Les cinq femmes de JMM se regardent vivre (ibid., 2015, p. 156) bien plus qu'elles ne vivent. Leur parole n'ayant aucune finalité performative, elles écoutent et contemplent l'expression verbale qu'elles donnent à leur vie. Si elles ont existé avant l'attente du Frère-Fils, elles ne découvriront pas une autre existence après cette attente, ni dans leur maison ni ailleurs. Les femmes sont les témoins très loquaces de leur propre vie. Le personnage "devient son propre objet ", et il ne parle que de luimême (Szondi, 1983 [1956], p. 64). En réduisant l'intersubjectivité, le texte dramatique accentue fortement l'auto-subjectivité. Les cinq femmes vivent, en commun, un 
métadrame intérieur dont la substance est l'interprétation de leur attitude d'attente, à partir des codes théâtraux et culturels qu'elles connaissent et qui ont cours.

\section{Une tragédie en moins}

JMM est aussi « un drame au second degré » (Sarrazac, 2015, p. 181). Les cinq femmes vivent le métadrame de n'avoir connu que le seul drame d'attendre le retour du FrèreFils. Aucun autre événement intense et douloureux n'a nourri leur vie; aucun événement terrifiant n'explique ou ne justifie que toutes ces vies, même les moins âgées, aient été faites de renoncement. Leur bagage existentiel ne contient qu'un seul fait qui n'a rien d'exceptionnel: un jeune homme, frère ou un fils, ou un petit-fils même, au terme de son adolescence, a quitté le toit familial pour devenir adulte et tenter l'aventure de la vie. Les cinq femmes, malgré tout, ont conscience de l'importance sans doute disproportionnée qu'elles ont donnée à ce fait, et des limites de leurs émotions. Elles examinent souvent leurs propres pensées, ou celles des autres, avec le recul d'un désengagement affectif : «Et dans ma tête, encore, je pensais cela : est-ce que je n'ai pas toujours attendu? et cela me fit sourire de me voir ainsi » (Lagarce, 1997, p. 7). Elles mesurent aussi combien le départ du Frère-Fils a rejoint l'univers des réalités fantasmées: "cela leur plaît aujourd'hui: des souvenirs de batailles rangées. Ont ${ }^{12}$ la belle imagination et fertile » (ibid., p. 42). Les femmes créent une distance énonciative vis-à-vis de leur propre univers, comme les déterminants et les pronoms démonstratifs en témoignent. Par exemple, elles se détachent de leur maison : «Je le regarde venir vers moi, vers moi et cette ${ }^{13}$ maison. Je le regarde » (ibid., p. 9). Elles introduisent également, contrairement à l'expression de leur sensibilité intense, une forte distance avec le Frère-Fils et à l'occasion avec les autres femmes : « lorsque le jeune frère celui-là » (ibid., p. 17), « celles-là », « et celles-là encore » (ibid., p. 23), « celle-là ta mère » (ibid., p. 34), « le jeune frère » (ibid., p. 43), « la mort de celui-là, la mort du jeune frère» (ibid., p.44). La famille semble composée de commentatrices qui s'examinent sans se sentir impliquées. De nombreuses expressions effacent leurs liens affectifs ; leur parole paraît extérieure à leur vécu et à leur intimité. Leur séparation énonciative suggère qu'elles ne participent pas pleinement au monde de leurs vies perdues, mais qu'elles y récitent des rôles qui ne leurs conviennent pas. Elles appartiennent à un événement qu'elles n'ont pas décidé intimement et dans lequel elles sont embarquées, semblables à des actrices en scène, en train de jouer une pièce qu'elles écrivent avec le regret d'y participer (ibid., p. 44) :

ce que nous faisons [...] on chante notre chanson, on danse notre danse un peu

lente, toutes les cinq,

toutes toujours comme nous nous avons toujours été,

comme nous avons toujours appris à le faire,

toutes ces années perdues,

notre pavane pour le jeune homme, cette histoire-là.

Les femmes commentent leur comportement avec la conscience qu'une actrice a de son personnage. Elles se considèrent, avec lucidité, comme des comédiennes qui jouent une pièce dans laquelle elles doivent représenter des personnages qui éprouvent une douleur intense provoquée par un faible malheur: "on voulait la tragédie, la belle famille tragique mais nous n'aurons pas cela, juste la mort d'un garçon dans une maison de filles [...] rien d'autre » (ibid., p. 45). Elles voudraient donner de l'intensité à leur histoire familiale, la faire entrer dans les histoires exemplaires : «on rêvait, on 
voudrait cela, on aurait voulu cela, ce serait beau et douloureux et noble encore... » (ibid., p. 45). Elles désireraient participer à un destin admirable, à l'héroïsme des sentiments familiaux absolus : "elle ne survécut pas à son frère, elle l'aimait tant qu'elle mourut avec lui, de détresse comme ça » (ibid.).

La mort du Frère-Fils hors scène ne produit pas une tragédie antique ni même une histoire contemporaine tragique, mais une plainte modulée à cinq voix, une mélopée des affects changeants au cours de cette attente. Attendre ce Frère-Fils a ligoté l'existence des femmes, mais aucune d'elle ne deviendra pour cela une héroïne. Celui qui revient n'a pas le prestige des voyageurs qui découvrent un nouveau monde, et celles qui sont restées n'ont pas l'aura des femmes qui se sont sacrifiées. C'est un monde qui ne donne que des rôles médiocres, sans gloire (Jongy, 2011, p. 9).

Les cinq femmes ne peuvent que constater l'abîme qui existe entre l'idée sublime qu'elles ont eue d'elles-mêmes et de leur situation, et la plate réalité dont elles ne sortent pas : "Elles se jouent à elles-mêmes la légende de leur vie " (Lagarce, 1997, p. 63). En fait, elles rêvent une vie qui serait un autre théâtre. La « légende » donnerait sens au malheur de l'attente, l'histoire familiale pourrait résonner sur la scène des vies admirables, et les cinq femmes seraient dignes du grand genre théâtral, la tragédie. Cette vision métathéâtrale laisse entendre que c'est la vie, pour être à la hauteur des existences attendues, qui devrait être une mimésis du théâtre. C'est dans le rapport à leur propre histoire, à leur propre situation, et à leur propre parole, que les cinq femmes déploient la théâtralité de JMM.

\section{Conclusion}

Le théâtre de J.-L.Lagarce, en particulier JMM, prolonge la dramaturgie de M. Maeterlinck et S. Beckett. Au plan de l'écriture, l'absence d'action se fonde sur un renouvellement du personnage dramatique, devenu aboulique, et une transformation de la parole dramatique. Les procédés linguistiques de J.-L. Lagarce dénaturalisent la parole en l'amplifiant. L'expression verbale déborde et prolifère, jusqu'à rompre avec la mimésis conversationnelle et métamorphoser totalement les usages de la parole authentique. Plus globalement encore, JMM, comme bien d'autres pièces en rupture avec l'aristotélisme, se montre en train de chercher une forme, voire un modèle. Il en résulte que la théâtralité est réinvestie par la métathéâtralité: la dramaturgie s'interroge sur son langage dramatique, et la situation énonciative fictionnelle du personnage est renvoyée à la situation énonciative du théâtre: le personnage se demande quel personnage de théâtre il est dans sa vie. Dès lors, la capacité de référence du texte dramatique au hors-texte et au hors-scène s'amenuise, la scène se replie sur elle-même et, d'une certaine manière, renforce le caractère "primaire » du drame (Szondi, 1983 [1956], pp. 16-17). 


\section{BIBLIOGRAPHIE}

ARISTOTE. (1980). La Poétique. Trad. du grec par R. Dupont-Roc \& J. Lallot. Paris : Éd. du Seuil. BECKETT, S. (1952). En attendant Godot. Paris : Éd. de Minuit.

BIDENT, C. (2010). «L'expérience du personnage ». Europe 969-970, p. 53-62.

Dessons, G. (2016). Maeterlinck, le théâtre du poème. Paris : Classiques Garnier.

Jolly, G. (2008). « La choralité ou la mise en mouvement de la parole ». In : Naugrette, C., \& Sarrazac, J.-P. (dirs), Jean-Luc Lagarce dans le mouvement dramatique, vol. IV, Colloque de Paris III. Besançon : Éd. Les Solitaires Intempestifs, p. 221-236.

JongY, B. (2011). « Introduction ». In : Les « Petites tragédies » de Jean-Luc Lagarce. Neuilly-les-Dijon : Éd. du Murmure, p. 7-16.

KunTZ, H. (2008), « Aux limites du dramatique ». In : Naugrette, C., \& Sarrazac, J.-P. (dirs), Jean-Luc Lagarce dans le mouvement dramatique, vol. Iv, Colloque de Paris III. Besançon : Les Solitaires Intempestifs, p. 11-28.

LAGARCE, J.-L. (1994). J'étais dans ma maison et j'attendais que la pluie vienne. Besançon : Éd. Les Solitaires Intempestifs.

LAGARCE, J.-L. (2011). Théâtre et pouvoir en occident. Besançon : Les Solitaires Intempestifs.

MAETERLINCK, M. (1917) [1896]. Le Trésor des humbles. Paris : Mercure de France.

MAETERLinCK, M. (2012) [1892]. Pelléas et Mélisande. Fédération Wallonie-Bruxelles : Espace nord.

MiRJoL, C. (2010). « L’oublié, tous les oubliés ». Europe 969-970, p. 63-76.

Pavis, P. (1980). Dictionnaire du théâtre. Paris : Éd. sociales.

SARRAZAC, J.-P. (2008). « De la parole du fils prodigue au drame-de-la-vie ». In : Naugrette, C., \& Sarrazac, J.-P. (dirs), Jean-Luc Lagarce dans le mouvement dramatique, vol. IV, Colloque de Paris III. Besançon : Les Solitaires Intempestifs, p. 271-296.

SARRAZAC, J.-P. (2012). Poétique du drame moderne. Paris : Éd. Le Seuil.

SARRAZAC, J.-P. (2015). Critique du théâtre 2. Strasbourg : Circé.

SzonDI, P. (1983) [1956]. Théorie du drame moderne. Trad. de l'allemand par P. Pavis avec la collaboration de J. \& M. Bollack. Lausanne : Éd. L'Age d'Homme.

TALBOT, A. (2008). «L'épanorthose : de la parole comme expérience du temps ». In : Naugrette, C., \& Sarrazac, J.-P. (dirs), Jean-Luc Lagarce dans le mouvement dramatique, vol. IV, Colloque de Paris III. Besançon : Les Solitaires Intempestifs, p. 255-270.

VALÉRO, J. (2008). « Diarisme et écriture dramatique : du journal à l'espace autobiographique ». In : Naugrette, C., \& Sarrazac, J.-P. (dirs), Jean-Luc Lagarce dans le mouvement dramatique, vol. IV, Colloque de Paris III. Besançon : Les Solitaires Intempestifs, p. 237-252. 


\section{NOTES}

1. "Enteschlussen » en allemand indique bien la décision qui est prise par un agent humain après une délibération ou un échange.

2. S. Beckett n'a pas droit à un chapitre dans le livre référence de P. Szondi (1983 [1956]), Théorie du drame moderne. P. Szondi justifie le choix des exemples, en 1963: "Cette étude a été écrite il y a une dizaine d'années. Le choix des exemples s'explique par cette date; il ne serait plus tout à fait le même si le livre devait être écrit aujourd'hui » (ibid., p. 136).

3. JMM dans la suite de l'article.

4. P. et M. dans la suite de l'article.

5. EAG dans la suite de l'article.

6. Ce personnage n'ayant pas de nom, nous l'appellerons le Frère-Fils, en reprenant la nature de ses principales relations familiales avec les cinq femmes de la pièce.

7. C'est nous qui soulignons.

8. Ce sont des images qui proviennent du fonds de la culture occidentale et qui sont chargées de connotations: rencontre dans une forêt, jeune personne inconnue, la descente dans les profondeurs du château, le lien entre la nature et le désir etc.

9. Dans la suite de la citation, tous les "Ils" représentent Pelléas et Mélisande.

10. Ni même par les gestes et le corps, mais ce n'est pas ici notre propos.

11. L. Pirandello par exemple.

12. Sic

13. C'est nous qui soulignons. Dans les citations suivantes aussi.

\section{RÉSUMÉS}

Les dramaturges dont les pièces, en rupture avec le modèle aristotélicien, sont sans action dramatique appartiennent à une généalogie désormais longue et fournie. Dans cet article, nous analysons J'étais dans ma maison et j'attendais que la pluie vienne de J.-L. Lagarce (1994) à la lumière de deux œuvres majeures qui lui ont ouvert la voie : Pelléas et Mélisande de M. Maeterlinck (1892) et En attendant Godot de S. Beckett (1952). Nous examinerons comment le texte prend forme sans aucun conflit structurant; nous regarderons quelles sont les conséquences de l'absence d'action sur le dialogue et la parole, et comment, au bout du compte, ces pièces, en particulier celle de J.L. Lagarce, s'inscrivent dans une théâtralité spécifique.

The playwrights whose plays, breaking with the Aristotelian model, are without dramatic action belong to a long and rich genealogy. This article analyses J.-L. Lagarce's (1994) J'étais dans ma maison et j'attendais que la pluie vienne in the light of two major works that paved the way for him: M. Maeterlinck's (1892) Pelléas et Mélisande and S. Beckett's (1952) En attendant Godot. We will examine how the text takes shape without any structuring conflict; we will look at the consequences of the absence of action on dialogue and speech, and how these plays, in particular that of J.-L. Lagarce, are part of a specific theatricality. 
INDEX

Mots-clés : action, conflit, dialogue, théâtralité

\section{AUTEUR}

JEAN-PAUL DUFIET

Università degli Studi di Trento, Dipartimento di Lettere e Filosofia, I- 38122 Trente, Italie 